\title{
Importancia de la estimulación temprana en niños menores de 5 años
}

\author{
Importance of early growth in kids
}

\author{
Sergio Hernández Rincón ${ }^{1}$, Luz A. Vizcaíno Pérez², Brenda J. Barrón Gómez ${ }^{3}$, Abril Q. Muñoz \\ $\operatorname{Veg} a^{4 *}$
}

\begin{abstract}
:
It is recommended that people (especially parents) keep in mind that early stimulation in children's preferable ages 0 - 5 years is applied, since the majority of brain development takes place until the child reaches 3 years, at the same While proliferate child neurons and synapses establishes new connections at high speed, children acquire the ability to think, talk, learn, reason, and established the foundations of social and biological behaviors that would mark it throughout adult life. During the first years of life, environmental factors and stimuli acquire great importance, if they are suitable, the nervous system will be held in better conditions. Since this prevents the wrong diagnosis or even late of diseases like mental retardation among others and help better coexistence and adaptation. By the above and considering that the test of evaluation of child development (EDI); It is the set of actions aimed at promoting physical, mental and social capabilities of the child, it is necessary to use in the prevention of delay in psychomotor development, diagnosing, curing and rehabilitating suitably for children to achieve the best development of the potentials of the individual, should be detected that your child has delayed their development.
\end{abstract}

\section{Keywords:}

Early stimulation, evaluation of child development, kids.

\section{Resumen:}

Es recomendable que las personas (especialmente padres de familia) tenga presente que la estimulación temprana en los niños de edades preferibles de 0-5 años sea aplicada, debido a que la mayor parte del desarrollo cerebral ocurre antes de que el niño cumpla 3 años, al mismo tiempo que las neuronas del niño proliferan y las sinapsis establecen nuevas conexiones a gran velocidad, los niños adquieren la capacidad de pensar, hablar, aprender, razonar y se establecen los fundamentos de los comportamientos sociales y biológicos que lo marcarían durante toda la vida adulta. Durante los primeros años de la vida, los factores ambientales y estímulos adquieren una gran importancia, si son adecuados, el sistema nervioso se desarrollará en mejores condiciones. ya que esto pre viene el mal diagnostico o incluso tardío de enfermedades como retraso mental entre otras, y ayude a una mejor convivencia y adaptación. Por lo anterior y considerando que la prueba de Evaluación del Desarrollo Infantil (EDI); Es el conjunto de acciones dirigidas a promover las capacidades físicas, mentales y sociales del niño, es necesario utilizarla en la prevención del retardo en el desarrollo psicomotor, diagnosticando, curando y rehabilitando oportunamente para los niños logren el mejor desarrollo de las potencialidades individuales, en caso de que se detecte que un niño presenta retraso en su desarrollo.

\section{Palabras Clave:}

Estimulación temprana, evaluación del desarrollo infantil, infantes.

\section{Desarrollo}

\subsection{Etapas de crecimiento y desarrollo}

El crecimiento y el desarrollo del niño son dos fenómenos íntimamente ligados. Sin embargo, conllevan diferencias que es importante precisar [1]. Se entiende por crecimiento un aumento progresivo de la masa corporal, tanto por el incremento del número de células como por su tamaño. El crecimiento conlleva un aumento del peso y de las dimensiones de todo el organismo y de las partes que lo conforman; se expresa en kilogramos y se mide en centímetros.

Este proceso se inicia en el momento de la concepción del ser humano y continúa a través de la gestación, la infancia, la niñez y la adolescencia. El crecimiento es inseparable del desarrollo y, por lo tanto, ambos están

${ }^{1}$ Lic. Médico Cirujano, Universidad Autónoma de Estado de Hidalgo, Escuela superior de Tepeji del Rio, Av. Del Maestro No. 41 Colonia Noxtongo $2^{\mathrm{a}}$ Sección, Tepeji del Rio, Hidalgo, México. Email: estre7216@hotmail.com, 2 luzvizcaino09@gmail.com, 3 jacquelinbarron_01@hotmail.com 4 abrilvega549@gmail.com 
afectados por factores genéticos y ambientales. El crecimiento físico de cada persona está sujeto a diversos factores condicionantes: factor genético, nutrición, función endocrina, entorno psicosocial, estado general de salud y afectividad.

El desarrollo implica la diferenciación y madurez de las células y se refiere a la adquisición de destrezas y habilidades en varias etapas de la vida.

El desarrollo está inserto en la cultura del ser humano. Es un proceso que indica cambio, diferenciación, desenvolvimiento y transformación gradual hacia mayores y más complejos niveles de organización, en aspectos como el biológico, el psicológico, el cognoscitivo, el nutricional, el ético, el sexual, el ecológico, el cultural y el social.

Hasta los 5 o 6 años de edad, las diferencias en el crecimiento dependen de la nutrición, del modo de alimentarse, del medio ambiente y de la atención sanitaria, más que de posibles factores genéticos o étnicos.

La importancia de este nuevo patrón radica en poder determinar si es adecuada la atención del niño con respecto a la nutrición, las necesidades básicas o la salud. De esta manera, se podrá detectar la obesidad, la desnutrición u otros trastornos infantiles de una manera rápida y temprana [2]

\subsection{Antecedentes de la estimulación temprana}

Las pruebas de evaluación del desarrollo favorecen la detección temprana de algunos trastornos como lo pueden ser: motrices, de lenguaje, posible retraso, encefalopatía hipóxico-isquémica, rasgos dismórficos/malformaciones, enfermedad de Steiner neonatal, distrofias musculares entre otras. Al mismo tiempo que permite brindar atención profiláctica a través de la estimulación temprana.

El término estimulación, que tiene sus orígenes en brindar estímulos al niño pequeño es tan viejo como el surgimiento de la humanidad ya que los estímulos en este ámbito pudieran considerarse todos aquellos que tienen un impacto sobre el ser humano que producen en él una reacción, es decir, una influencia sobre alguna función y que pueden ser de diferentes índoles externas, internas, algunas físicas, otras afectivas.

La relación del niño con los estímulos del medio debe darse en un intercambio estricto, de ahí la necesidad de una planificación estricta y no dejarla a la espontaneidad. La estimulación temprana (ET) surge simultáneamente en Estados Unidos $\mathrm{Y}$ toma el nombre de Estimulación Precoz.

Hoy en día se tiene un conocimiento más acerca de la existencia de un periodo sensitivo del desarrollo humano que ocurre solo en las etapas iniciales de la vida, por ello surgió la necesidad de proporcionar la estimulación adecuada al momento oportuno en los menores. Aunque se dice que la estimulación más importante es la que va desde los 0 a los 3 años. Se ha demostrado que es beneficioso continuar hasta los seis años, etapa en la que se "estimula al desarrollo".

\subsection{Historia de la prueba EDI en México}

En México, no se contaba con una prueba de evaluación del desarrollo infantil con propiedades psicométricas. La prueba de evaluación del desarrollo infantil (EDI) se desarrolló con este fin. El objetivo de este trabajo fue determinar las propiedades psicométricas de la EDI como prueba de tamizaje para los problemas de desarrollo infantil en menores de 5 años. Métodos. Se realizó un estudio transversal que incluyó pacientes menores de 5 años en tres entidades de la República Mexicana: Chihuahua, Yucatán y Distrito Federal. El espectro de la población incluyó niños con factores de riesgo biológico, ambiental y sin riesgo para retraso en el desarrollo. Se excluyeron los pacientes con alteraciones neurológicas evidentes. Se consideró, como prueba diagnóstica, el Inventario de Desarrollo de Battelle-2 en las tres entidades. En el Distrito Federal, adicionalmente, se aplicó Bayley [3].

En el Plan Nacional de Desarrollo 2013-2018 del Gobierno de la Republica, en la meta nacional: México incluyente, existen múltiples líneas de acción directamente Relacionadas con el Desarrollo Infantil, en el cual se garantiza el ejercicio efectivo de los derechos sociales para toda la población, fortaleciendo el desarrollo de capacidades en los hogares con carencias para contribuirá mejorar la calidad de vida e incrementar su capacidad reproductiva [4].

\section{Conclusiones}

En la sociedad especialmente en los padres de familia debe ser necesario el tener conocimiento sobre la estimulación temprana en niños menores de 5 años, esta práctica es fundamental para el desarrollo mental en cuanto a pensamiento, aprendizaje y razonamiento del menor y al igual al desarrollo físico. Esta práctica tiene como finalidad ayudar a identificar o prevenir alguna enfermedad cerebral o retraso psicomotor ayudando al niño a tener una mejor convivencia y adaptación con su ambiente físico; así como, optimizar el desarrollo del niño normal.

\section{Agradecimientos}

Agradecemos la gran colaboración de Berenice Reséndiz Serrano en investigación realizada y quién sigue apoyando de una manera externa.

\section{Bibliografía}


[1] (M, 2013, págs. 1-5)

[2] (Santrock, 2006, págs. 1-20)

[3] (Unidad de Investigación en Neurodesarrollo (UIN) , 2013)

[4] (Guadarrama Orozco, 2018, págs. 2-32)

Haga clic o pulse aquí para escribir texto. 